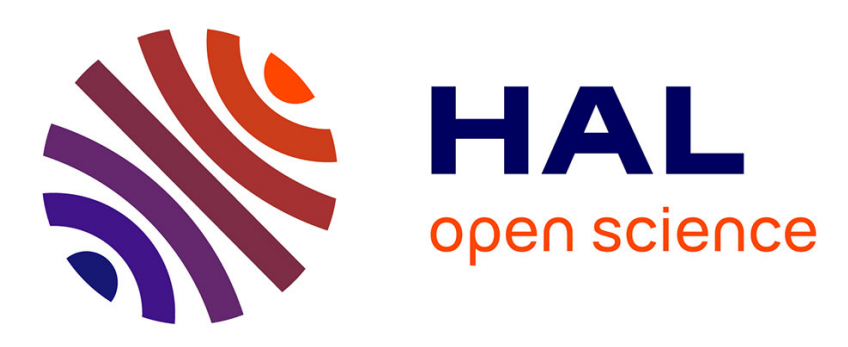

\title{
Looking for Mimicry in a Snake Assemblage Using Deep Learning
}

Thomas de Solan, Julien Renoult, Philippe Geniez, Patrice David, Pierre-André Crochet

\section{- To cite this version:}

Thomas de Solan, Julien Renoult, Philippe Geniez, Patrice David, Pierre-André Crochet. Looking for Mimicry in a Snake Assemblage Using Deep Learning. American Naturalist, 2020, 10.5061/dryad.sj3tx961q . hal-03021754

\section{HAL Id: hal-03021754 \\ https://hal.science/hal-03021754}

Submitted on 24 Nov 2020

HAL is a multi-disciplinary open access archive for the deposit and dissemination of scientific research documents, whether they are published or not. The documents may come from teaching and research institutions in France or abroad, or from public or private research centers.
L'archive ouverte pluridisciplinaire HAL, est destinée au dépôt et à la diffusion de documents scientifiques de niveau recherche, publiés ou non, émanant des établissements d'enseignement et de recherche français ou étrangers, des laboratoires publics ou privés. 


\title{
Looking for Mimicry in a Snake Assemblage Using Deep Learning
}

\author{
Thomas de Solan, ${ }^{*}$ Julien Pierre Renoult, Philippe Geniez, Patrice David, ${ }^{\dagger}$ \\ and Pierre-André Crochet ${ }^{\dagger}$
}

Centre d'Ecologie Fonctionnelle et Evolutive, Université de Montpellier, CNRS, École Pratique des Hautes Études-Paris Science et Lettres, Université Paul Valéry Montpellier 3, Institut de Recherche pour le Développement, Montpellier, France

Submitted July 1, 2019; Accepted November 20, 2019; Electronically published May 27, 2020

Online enhancements: appendix, R code. Dryad data: https://doi.org/10.5061/dryad.sj3tx961q.

\begin{abstract}
Batesian mimicry is a canonical example of evolution by natural selection, popularized by highly colorful species resembling unrelated models with astonishing precision. However, Batesian mimicry could also occur in inconspicuous species and rely on subtle resemblance. Although potentially widespread, such instances have been rarely investigated, such that the real frequency of Batesian mimicry has remained largely unknown. To fill this gap, we developed a new approach using deep learning to quantify the visual resemblance between putative mimics and models from photographs. We applied this method to Western Palearctic snakes. Potential nonvenomous mimics were revealed by an excess of resemblance to sympatric venomous snakes compared with random expectations. We found that $8 \%$ of the nonvenomous species were potential mimics, although they resembled their models imperfectly. This study is the first to quantify the frequency of Batesian mimicry in a whole community of vertebrates, and it shows that even concealed species can act as potential models. Our approach should prove useful for detecting mimicry in other communities, and more generally it highlights the benefits of deep learning for quantitative studies of phenotypic resemblance.
\end{abstract}

Keywords: imperfect mimicry, camouflage, deep neural network, Batesian mimicry.

\section{Introduction}

Batesian mimicry occurs when palatable or harmless species evolve to resemble an unpalatable or dangerous model species and consequently gain protection from predators (Bates 1862). Mimicry is a canonical example of evolution through natural selection; it was already discussed by Darwin with Wallace (Darwin 1887) and still constitutes a proliferous field of research (Sherratt 2002; Nishikawa

\footnotetext{
* Corresponding author; email: thomas.de-solan@cefe.cnrs.fr.

$\dagger$ Co-senior authors.

ORCIDs: de Solan, https://orcid.org/0000-0001-5872-566X.
}

Am. Nat. 2020. Vol. 196, pp. 74-86. (C) 2020 by The University of Chicago. 0003-0147/2020/19601-59346\$15.00. All rights reserved.

DOI: $10.1086 / 708763$ et al. 2015; Joshi et al. 2017; Bosque et al. 2018). Nevertheless, some fundamental aspects of this defensive strategy remain poorly known - notably its frequency in plants and animals - mainly because detecting mimicry is no simple matter.

Research on Batesian visual mimicry has long been confined to a few spectacular cases, such as hoverflies (Penney et al. 2012), butterflies (Kitamura and Imafuku 2015), and kingsnakes (Brodie and Janzen 1995), that possess conspicuous colors and show a resemblance to their models so striking that it cannot be fortuitous. This perfection is a double-edged sword: it served as evidence for Darwinian evolution, but it also provided a distorted picture of what mimicry can be. Indeed, neither conspicuousness nor extreme resemblance is a necessary condition for Batesian visual mimicry. Even inconspicuous or camouflaged venomous species may be spotted by predators that can recognize them by using cues that have not necessarily evolved as signals. Harmless and palatable species can mimic these cues to gain additional protection from mimicry while being themselves cryptic (Barlow and Wiens 1977; Mound and Reynaud 2005; Gianoli and Carrasco-Urra 2014; Corcobado et al. 2016). Last, experimental evidence suggests that imperfect resemblance between mimics and models, known as imperfect mimicry, can provide protection against predators (Dittrich et al. 1993). Focusing on canonical examples of striking mimicry using conspicuous signals is thus likely to bias our perception of mimicry and underestimate its frequency.

The detection of visual mimicry within a community requires a quantification of the resemblance between species. In mimicry studies two main categories of methods have been traditionally used to estimate resemblance. The methods in the first category analyze predefined features in images - for example, morphometric measures, the geometry of color patterns, or the distribution of orientations and spatial frequencies in light changes (Penney 
et al. 2012; Taylor et al. 2016; Troscianko et al. 2017; Endler et al. 2018). These methods usually attempt to model physiological perceptual processes and can thus provide information on the role of these processes in the evolution of mimicry. However, they may be unable to capture the high dimensionality of the feature space relevant for assessing visual resemblance (Zhang et al. 2018). The second category of methods relies on psychological tests with human subjects (e.g., Cuthill and Bennett 1993). These methods can provide more accurate assessments of overall perceptual resemblance; however, extensive tests and very large sample sizes are necessary to buffer the individual variations between evaluators (Ishihara et al. 2001; Kitayama et al. 2003).

Artificial intelligence, and especially deep convolutional neural networks (DCNNs), offers new opportunities to model perceptual resemblance (Jozwik et al. 2017; Cichy and Kaiser 2019). DCNNs are a class of artificial neural networks composed by a large number $(>50)$ of learnable filters (hence the "deep") and which use a convolution (i.e., a mathematical operation on two functions that produces a third function expressing how the shape of one is modified by the other) to reencode the data with which they are fed (Goodfellow et al. 2016). A psychophysical experiment conducted with thousands of humans showed that measures of resemblance between complex stimuli (e.g., pictures of a crowd) by DCNNs predict the average humanperceived resemblance better than any previous models (Zhang et al. 2018). Moreover, DCNNs can estimate resemblance despite noise arising from differences in position, orientation, occlusion, and illumination among images (Jozwik et al. 2017). Thus, DCNNs are highly suited to exploit the nearly inexhaustible resource of unstandardized photographs posted on the World Wide Web. DCNNs have revolutionized many scientific fields in recent years, including biology (e.g., genomics [Zhou and Troyanskaya 2015] and medicine [Shen et al. 2017]), but the potential of DCNNs to act as models of human and animal perception has been overlooked in visual ecology thus far.

Several cases of Batesian visual mimicry between venomous and nonvenomous snakes of the Western Palearctic (Europe, North Africa, and the Middle East) have been reported in the literature, sometimes on the basis of experimental or behavioral data but mainly using a human subjective assessment of the visual resemblance between potential model and mimic (Gans 1961; Werner and Frankenberg 1982; Werner 1983; Valkonen et al. 2011b; Aubret and Mangin 2014; Valkonen and Mappes 2014). None of the venomous species in the study area possesses highly conspicuous colors, and most of them are cryptic in their environment (Turk 1958; Heatwole and Davison 1976; Werner 1983). However, the zigzag dorsal patterns of vipers have been shown to elicit avoidance in bird pred- ators (Valkonen et al. 2011a) in addition to providing camouflage (Santos et al. 2014). In spite of these case studies, no attempt has been made to evaluate the extent of mimicry in the Western Palearctic snake assemblage. Western Palearctic snakes thus appear as a good model to measure the frequency of mimicry in situations that are more complicated than most classic examples described in the literature. Here, we incorporated DCNNs into a workflow to identify potential mimics among all of the 122 snake species known in the Western Palearctic at the time of the study.

To identify candidate mimics, we developed a DCNN method to quantify visual resemblance and combined this information with geographic distributions. We classified as a potential mimic any nonvenomous species that met two criteria: (i) its resemblance with (at least) one of the venomous species of the West Palearctic was particularly high and (ii) the degree of sympatry with the most resembling venomous species was higher than that expected from a random draw. The literature mentions several species in our assemblage that may be mimetic; we did not incorporate this as prior information but rather posteriorly compared our results with the literature.

\section{Material and Methods}

We analyzed all 122 snake species of the Western Palearctic (according to Geniez 2015, with the additional species described since), including 35 venomous species and 87 nonvenomous species (see table A1, available online, for information on venomousness, the number of images analyzed, and the source of distribution data).

\section{Image Data Sets}

We created two image data sets: one for the nonvenomous snakes ( 838 images; mean $=9.6$ images per species) and one for the venomous snakes (1,802 images; mean = 50.0 images per species; details are provided in table A1). All venomous species exhibit some level of intraspecific variation, which can be geographically structured (and often formally recognized as different subspecies) or present in most populations as individual variation. Moreover, some species exhibit distinct morphotypes that can be easily identified by humans (for examples of different morphotypes in Vipera aspis, see fig. A1; figs. A1-A5 are available online). We did not treat these conspecific morphotypes as distinct classes. As DCNNs can solve nonlinear problems, we expected the networks to learn that these different morphotypes belong to a single species. However, in general, classes with high variation are more difficult to recognize for DCNNs (e.g., Fuentes et al. 2018); 
we thus used more images for venomous species with several morphotypes (up to 84 images per species; see table A1). All images represent different individuals except for a few species for which only a few images were available.

In addition to the 35 species of Western Palearctic venomous snakes (each representing a distinct class of pictures), we added one class of "foreign" venomous snakes to the venomous data set. This class was made of 80 photographs of 80 different species living outside the Western Palearctic but in climates similar to those of the study area (i.e., temperate and desert; see the list of species used in table A1)

We selected only images showing a clear dorsal view of the whole specimen (see fig. A1 for examples). We collected $80 \%$ of the images from the internet, with the remaining taken in the field by us or colleagues. An expert herpetologist (P. Geniez) validated all identifications individually. Because images are rare for several of the studied species, our data sets may appear small compared with those of other studies using DCNNs. However, recent investigations have revealed that with appropriate training (see "transfer learning" in "DCNN Training"), DCNNs can reach very high performances even when trained on limited data sets (Körschens et al. 2018). This is confirmed by our results (see below).

\section{Geographic Distribution Data Set}

We constructed distribution ranges from three types of data: (1) when available, we used distribution maps downloaded from the IUCN Red List website (http://www .iucnredlist.org, accessed February 2018); (2) otherwise, we reconstructed by hand the spatialized distribution maps from pictures of distribution ranges found in the World Health Organization's database of venomous snakes (http:// apps.who.int/bloodproducts/snakeantivenoms/database/) and from other specialized literature; and (3) when no distribution map was available, we built maps from occurrence data found in the specialized literature (references are listed in table A1). We used QGIS version 2.18 (Quantum GIS Development Team 2013) to create the distribution maps. Each distribution map was depicted by a spatialized polygon projected in the World Geodetic System (WGS 84).

\section{Methodological Workflow}

DCNNs were first trained to recognize the different venomous species of the Western Palearctic and of the foreign class (fig. 1A). Trained DCNNs were then asked to classify pictures of nonvenomous snakes as one of the venomous species or as belonging to the foreign category (fig. $1 B$ ). For each image, DCNNs provided a probability of being attributed to each of these classes. This probability is not an absolute measure of resemblance but instead is a measure of the relative resemblance with a model compared with the resemblance with other models. We added the foreign class to limit the risk of picking up a "most resembling venomous snake" even if the image does not particularly resemble any of the venomous snakes (Dalyac et al. 2014). The foreign class, which groups together a high diversity of patterns, is expected to act as an attractor for such nonvenomous species. Thus, we are confident that most nonvenomous species consistently identified by the DCNNs as one of the nonforeign venomous species do show a true resemblance.

The mean attribution probability (averaged across all available pictures) of each nonvenomous species to each venomous species was then used to build a matrix of resemblance between nonvenomous and venomous species. These probabilities were transformed into resemblance scores (i.e., in inversed ranks of probability; $1 / \mathrm{rank}$, in ascending order; fig. 1C). Thus, the class of venomous snakes to which one particular nonvenomous snake was attributed most often (i.e., ranking first or second) had the highest resemblance score. This step allowed us to reduce the prediction variability arising from the use of different training algorithms (Sameen and Pradhan 2017; Kim et al. 2019) and prevented overconfident predictions (Ju et al. 2018). It also increased the match between the measure of resemblance by DCNNs and the perceptual space of animals (for work on humans, see Zhang et al. 2018). Next, we deleted from this matrix the foreign venomous species class and all nonvenomous species for which the foreign class received the highest resemblance score, thus discarding species that were clearly not mimics (60 nonvenomous species discarded). In parallel we built a sympatry matrix the same size as the resemblance matrix that gives the proportion of the geographic distribution of each nonvenomous species that overlaps with the distribution of each venomous species. Following a previous study (Miller et al. 2019), we considered that the proportion of sympatry reflected the potential extent of selection for mimicry. Even if a few allopatric species pairs have distribution ranges close enough to allow predators to associate both snake species, most allopatric pairs are separated by hundreds or thousands of kilometers, which limits this possibility (Pfennig and Mullen 2010). The last step of our workflow was to calculate total scores for each nonvenomous species by summing the resemblance scores weighted by sympatry scores (Hadamard matrix product).

Candidate mimics and models were identified statistically by comparing total scores with a null distribution of randomized total scores. This null distribution was generated by randomly shuffling the venomous species in the sympatry matrix before weighting the resemblance score. 


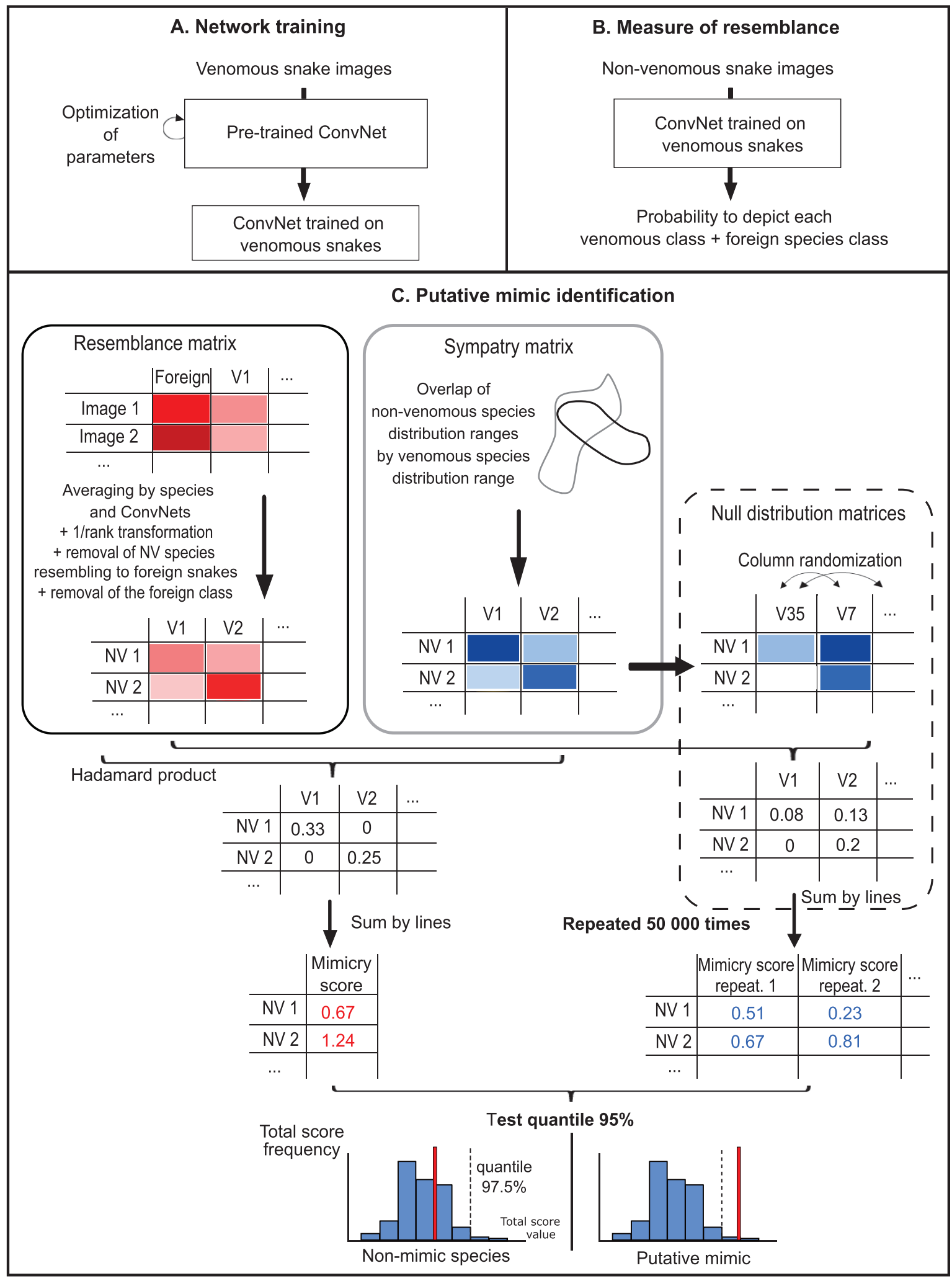

Figure 1: Workflow of the three-step method used to detect candidate mimic species among nonvenomous snakes and candidate model species among venomous ones. A, Training of the artificial neural networks to recognize 35 venomous snake species and the foreign venomous snake class. $B$, Measuring the resemblance of nonvenomous snakes (one measure per image) with each species of venomous snakes and the foreign class. $C$, Building the resemblance matrix and the sympatry matrix and testing an excess of resemblance between sympatric species using a null distribution to identify candidate mimics. ConvNet $=$ convolutional neural network; $\mathrm{NV}=$ nonvenomous; $\mathrm{V}=$ venomous. 
The weighted resemblance scores were then summed for each nonvenomous species to obtain the randomized total score. We replicated this operation 50,000 times (fig. 1C). Species for which the total scores fell in the right tail (e.g., the $5 \%$ highest scores) of the null distribution were more similar to sympatric venomous species than expected by chance and were thus considered candidate mimics.

Since we tested the same hypothesis of an excess of resemblance multiple times, the chance of false discovery (i.e., a significant result arising by chance) also increased. We used Fisher's method, also named Fisher's combined probability test (Fisher 1925), to test whether the $P$ values of the individual mimicry tests (estimated as the proportion of randomized scores higher than or equal to the real score) were distributed according to a uniform distribution or presented an excess of low values, indicating a significant excess of resemblance with the sympatric venomous snakes. We combined the $P$ values of the 27 nonvenomous species (out of 87 ) that were not eliminated by being classified in the foreign class into a $\chi^{2}$ statistic and compared it with a $\chi^{2}$ distribution with $2 \times 27$ degrees of freedom. This effectively provides a global test of the hypothesis that mimicry affected the evolution of phenotypes in the Western Palearctic snake assemblage. All statistical analyses were performed with R ( $\mathrm{R}$ Development Core Team 2008).

Our method did not include phylogenetic correction for the measure of the resemblance because all of the venomous and nonvenomous snakes belong to two distant and monophyletic clades. The resemblance between these clades thus cannot be attributed to phylogenetic relationship (see "Discussion").

\section{DCNN Training}

Training a DCNN to recognize snake species from scratch would have required a very large data set of images to achieve good performances. Following other studies (e.g., Norouzzadeh et al. 2018), we used "transfer learning," in which a DCNN pretrained on a very large data set is finetuned with a new, smaller data set. All details about the structure and the training of DCNNs are available in the appendix (available online). All code is available online, in a zip file, as well as at https:/github.com/t-desolan/Mimicry _snakes. ${ }^{1}$ The images used for training and testing can be provided on request.

\section{Test of Background Influence}

To confirm that network predictions were made on the basis of the snakes and not on the background, snakes were

1. Code that appears in The American Naturalist is provided as a convenience to readers. It has not necessarily been tested as part of peer review. cut out in 60 photos and pasted onto photos of ground only. Ground photos were similar to backgrounds found in snake photos and depicted ground with rocks, dead leaves, plants, or sand. Of the 60 snake images, 20 belonged to venomous snakes, 20 belonged to nonvenomous snakes previously identified as mimetic, and 20 belonged to nonvenomous snakes previously identified as nonmimetic. Then we used nonparametric multivariate analysis of variance (MANOVA) with the adonis function of the vegan package (Dixon 2003) in R to test the effect of background replacement on resemblance scores. We controlled variation between species by entering species names as an explanatory variable. We checked for multivariate homoscedasticity using the betadisper function of the vegan package (nonsignificant, effect of background: $F=0.15$, $P=.69)$.

\section{Results}

After training, DCNNs were able to recognize the species of a Western Palearctic venomous snake with an accuracy of $77 \% \pm 0.004 \%$ SE (percentage of pictures correctly classified, evaluated on the validation data set; random guess: $2.78 \%$ accuracy) and a top-three accuracy of $92 \% \pm 0.015 \%$ SE (probability that one of the three classes with the highest prediction matches the correct class), indicating that DCNNs learned the most distinctive traits of each species (precision per class is detailed in fig. A2).

Comparing weighted resemblance scores with the null distribution, with a $5 \%$ threshold, our method identified seven nonvenomous species out of the $87(8 \%)$ as candidate mimics of venomous snakes: these species have a higher resemblance with their sympatric venomous species than with a random assemblage of venomous snakes. A list of potential mimics and models is given in figure 2, with more details provided in figure A3. Fisher's method confirmed the overall significance of excessive resemblance with sympatric venomous snakes across the tested nonvenomous species set $\left(\chi^{2}=109.3, P<.001\right)$. In addition, resemblance scores were not driven by similarity between image backgrounds, as they were not affected by shuffling backgrounds among images (nonparametric MANOVA performed with 60 images; background effect: $F=0.74, P=.67)$.

\section{Discussion}

As far as we know, we provide here the first attempt to quantify the extent of mimicry in an assemblage of vertebrates at a continental scale (and we know of only one similar study in invertebrates; Wilson et al. 2015). Although the hypothesis of mimicry would have to be validated experimentally for each particular species pair, our 

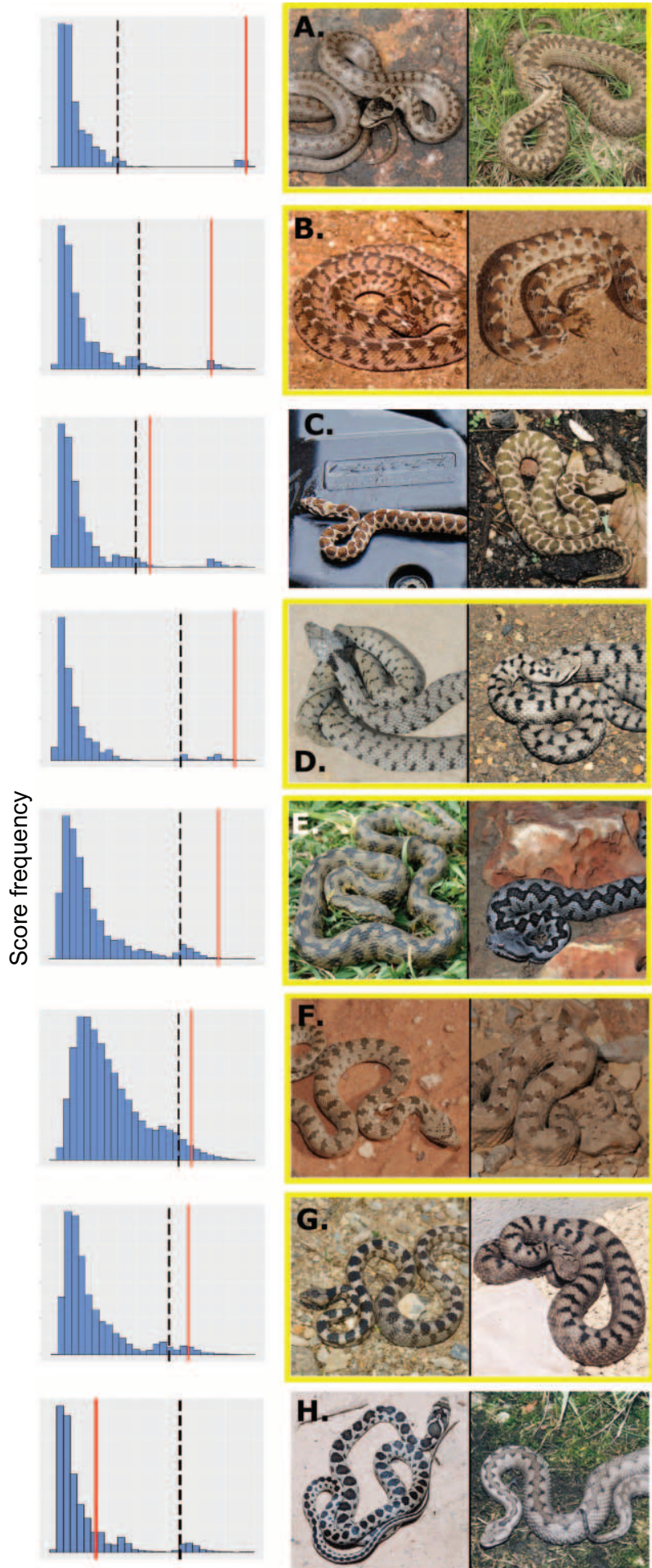

Score value

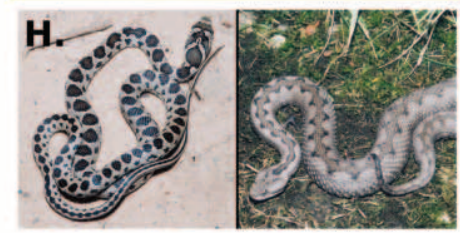

Figure 2: The seven mimic and model species $(A-G)$ identified in our analysis and one example pair of sympatric but nonresembling species $(H)$, with mimicry scores (red), 95\% quantiles (black dashed line), and the null distribution (blue) of each species pair. Species framed in yellow have been previously reported as mimetic in the analysis indicates the existence in Western Palearctic snakes of a pattern typical of Batesian mimicry (i.e., unusually high resemblance between sympatric pairs of nontoxic and toxic preys) that influenced the phenotypic evolution of this assemblage. The visual comparison of models and mimics suggests that their phenotypic resemblance is based on coloration and pattern (figs. 2, A2).

Venomous and nonvenomous species of snakes in our community belong to different families, so relatedness cannot explain this pattern, and mimicry itself probably evolved at least six times, as all but two of the candidate mimetic species belong to different genera (the exceptions are the two Natrix species; for the phylogenetic trees of the venomous and nonvenomous species, see fig. A4).

\section{Are Our Candidate Mimic Species Really Mimicking Venomous Snakes?}

Several arguments from the natural history of snakes support the validity of our method to identify mimics and models. First, six of the seven species identified as potential mimics by our method have been mentioned as such in the literature (Gans 1961; Werner and Frankenberg 1982; Werner 1983; Valkonen et al. 2011b; Aubret and Mangin 2014; see fig. 2). Second, the mimicry hypothesis is supported for all of the detected species by additional evidence based on behavioral and/or acoustic traits. The putative mimics Natrix helvetica, Natrix maura, Coronella austriaca, Spalerosophis diadema, and Telescopus fallax displace their maxillary bones to produce a triangular head shape when disturbed, increasing visual resemblance with their viper model (Werner and Frankenberg 1982; Werner 1983; Valkonen et al. 2011b; Aubret and Mangin 2014). In addition, N. maura also produces a hissing sound similar to that of the sympatric Vipera aspis (Aubret and Mangin 2014), while the nonvenomous Dasypeltis sahelensis and its sympatric venomous Echis pyramidum possess the same modified scales on the flanks that are rubbed to produce a similar warning signal when disturbed (Gans 1961). Finally, even if it has not been reported yet as a potential mimic in the literature, Hemorrhois nummifer

literature (Gans 1961; Werner and Frankenberg 1982; Werner 1983; Valkonen et al. 2011b; Aubret and Mangin 2014). A, Coronella austriaca and Vipera berus $(P=.004) . B$, Dasypeltis sahelensis and Echis pyramidum $(P=.02)$. C, Hemorrhois nummifer and Montivipera xanthina $(P=.04) . D$, Natrix helvetica and Vipera aspis $(P=.003)$. E, Natrix maura and Vipera latastei $(P=.004) . F$, Spalerosophis diadema and Pseudocerastes fieldi $(P=.02) . G$, Telescopus fallax and Vipera ammodytes $(P=.02)$. $H$, Hemorrhois hippocrepis and $V$. latastei $(P=.18)$. Photo credits: Matthieu Berroneau, Olivier Buisson, Luis García-Cardenete, Alexandre Cluchier, Philippe Geniez, André Langenbach, Jean Muratet, Gilles Pottier, and Jean-François Trape. 
shows the same behavioral and acoustic warning signal as the sympatric vipers Montivipera xanthina and Macrovipera lebetina (P.-A. Crochet and P. Geniez, personal observation).

Although additional cues support the mimicry hypothesis for all detected species, our method can only suggest candidate mimics, and a proper demonstration of mimicry would require experimental data. Indeed, most studies of interspecific resemblance are fundamentally limited in their ability to distinguish between mimicry and other factors of resemblance. Resemblance could arise by ecological convergence because of similar abiotic environmental constraints or similar selective pressures for camouflage (Rajpurohit et al. 2008; Stevens et al. 2013). These factors could lead to overestimating the frequency of mimicry in our results. This issue potentially affects all mimicry studies that do not test predator behavior in a natural environment. However, because most studies focus on conspicuously colored and presumably aposematic species (Brodie and Janzen 1995; Cheney and Marshall 2009; Penney et al. 2012), researchers usually assume that resemblance can be attributed only to mimicry. This assumption is seldom tested even if conspicuous colors may have a camouflage function (Mochida et al. 2015; Barnett et al. 2018). In Western Palearctic snakes, body colors are not conspicuous. It would thus be interesting to perform predation tests in natural conditions to investigate whether the features copied by potential mimics are those effectively used by predators to avoid models (e.g., as in Kristiansen et al. 2018). Nevertheless, as discussed previously, all identified mimics use behavioral or acoustic imitation in addition to color pattern, and as resemblance in several ontogenetically independent traits can hardly be attributed to ecological convergence, mimicry remains the most likely explanation in our view.

\section{DCNNs Can Identify Imperfect Mimicry of Camouflaged Models}

Remarkably, we found that even well-camouflaged species (e.g., vipers of the genus Pseudocerastes; fig. 2) could be a model for nonvenomous species. The lack of any pattern or color signaling venomousness in this species confirms that mimicry can be effective without conspicuous traits and advocates for a better separation between the concepts of Batesian mimicry, communication signaling, and conspicuousness. The link between these three concepts seems to represent a historical legacy of the easier detection of mimicry when it involves conspicuous aposematic models. The method presented here, which uses DCNN-based resemblance coupled with information on geographic co-occurrence, avoids a biased selection favoring conspicuous organisms when studying Batesian mimicry.

An additional advantage of our DCNN-based approach is its ability to suggest mimicry even when resemblance is imperfect. Most of the species identified by our method are imperfect mimics, at least from our human point of view, as their colors and patterns only partially match those of their model (fig. 2). Although any of the 11 nonexclusive hypotheses proposed in the literature to explain imperfect mimicry (for a detailed list, see Kikuchi and Pfennig 2013; Dalziell and Welbergen 2016) could apply to Western Palearctic snakes, only the "backup signal" hypothesis is currently supported by ecological evidence. This hypothesis suggests that imperfect mimicry can be reinforced by complementary signals (Johnstone 1996). It is convincing especially for mimics that show similarities in behavioral, morphological, and acoustic warning signals with their sympatric venomous species (Gans 1961; Werner 1983; Valkonen et al. 2011b; Aubret and Mangin 2014). In addition, the variability of models could help to maintain imperfect mimicry: it may prevent predators from associating a specific phenotype to venomousness (Kikuchi and Pfennig 2010) and thus make them more careful and less likely to attack snakes with imperfectly matching phenotypes. This explanation is plausible for at least the three candidate models Vipera ammodytes, Vipera berus, and V. aspis, which exhibit significant intraspecific and often intrapopulation variation in coloration (fig. A1). Finally, mimicry is a matter of receiver perception. A poor mimicry for our vision system may be better for another vision system. The different snakes' predators are likely to display different visual systems (e.g., bird and mammals), each shaping the evolution of mimicry in different ways.

\section{Performance of the Method to Identify Putative Mimics}

The ability to detect imperfect and nonconspicuous mimicry makes our method less conservative compared with methods based entirely on human detection for evaluating the frequency of mimicry. Even so, we may have failed to identify potential mimic-model pairs. First, the 5 th percentile threshold in the null distribution used to interpret resemblance scores is entirely conventional and may overlook some mimicry systems. We found two species below but close to the 5 th percentile threshold. The first species, Dolichophis jugularis, displays the same black coloration as its sympatric species, Walterinnesia morgani, but it has a different timing of activity (one is diurnal, while the other is nocturnal) and is thus probably not a mimic. For the second species, Rhagerhis moilensis, coloration mimicry of the sympatric species, Cerastes cerastes, 
cannot be discarded and should be subjected to further examination. Therefore, the 5th percentile threshold should be interpreted cautiously, and we recommend not drawing firm conclusions without examining species close to a decision threshold. Second, we may have failed to identify candidate mimics and models because we could not test mimicry for each morphotype separately for the nonvenomous polymorphic species (e.g., Leviton 1970; Kark et al. 1997; Mebert 2011). Instead, we had to analyze all morphotypes together and test mimicry at the species level. This complicates the detection of mimicry, as only some morphotypes of these polymorphic species may mimic venomous snakes (e.g., in Hemorrhois ravergieri; Werner and Frankenberg 1982; see below). Testing mimicry for each morphotype would require mapping the geographical distribution of each morphotype known, but this information was not available for most polymorphic species. Third, DCNNs cannot detect resemblance from information not present in the data: mimicry attributable to ultraviolet (UV) coloration (see next paragraph), behavior, acoustic, or olfactive signals will not be identified. These three limits may lead to an underestimation of the mimicry frequency in natural communities.

Among the species suggested as mimetic in the literature, three were not identified in our study (Gans 1961; Werner and Frankenberg 1982; Werner 1983). One of them, $H$. ravergieri, is considered a mimic of the sympatric Vipera ammodytes transcaucasiana (Werner and Frankenberg 1982). Our method failed to identify it as such, probably because it exhibits a strong polymorphism, with only some of its variants thought to mimic vipers (Werner and Frankenberg 1982). A second species, Dasypeltis bazi, could have been missed by our approach because of the limited number of pictures in our data set (six in total; this rare and poorly known species was recently described by Saleh and Sarhan [2016]) and the marked variation in coloration between individuals. However, the third unrecognized mimic, Telescopus dhara (Werner 1983), is not polymorphic, and we believe that claims for mimicry in this species are too optimistic, as it bears no particular resemblance with the proposed model Echis coloratus from visual inspection.

\section{Use of DCNNs to Assess Phenotypic Resemblance}

Several lines of evidence indicate that our method provides an accurate estimation of resemblance. First, the performance of DCNNs in image classification was remarkably high. It is similar or superior to that of other studies (e.g., Carranza-Rojas et al. 2017; Van Horn et al. 2018) despite the limited size of the training data set and the extreme resemblance among several vipers that makes their identification challenging when the geographic origin is unknown, even for experts (Geniez 2015). In comparison, a previous DCNN-based analysis of species recognition using 579,184 unstandardized training images representing 5,098 species reached $68.5 \%$ and $88.2 \%$ topone and top-five accuracy, respectively (Van Horn et al. 2018). Thus, our analysis confirms previous research by demonstrating that DCNNs can reach a high level of accuracy even when trained on small data sets (Hansen et al. 2018; Körschens et al. 2018; Mathis et al. 2018). Second, we avoided by-default classification, as more than twothirds of the nonvenomous species were identified as the foreign species class and removed from further analysis. These results support the idea that DCNNs can be effective tools for measuring phenotypic resemblance.

Our study highlights the benefits of using DCNNs to study resemblance between phenotypes in ecology and evolution. DCNNs have been previously used in ecology to analyze big data (e.g., to identify species from camera trap images; Norouzzadeh et al. 2018). However, their capacity to model biological perception has been thus far overlooked (Cichy and Kaiser 2019). A growing body of literature on humans has shown that DCNNs predict the perceived resemblance between complex stimuli better than other models, even those specifically tuned with neurophysiological data (Yamins et al. 2014; Kriegeskorte 2015; Kubilius et al. 2016). Of particular relevance for the study of camouflage and mimicry, one study showed that whichever perceptual task a DCNN is trained for (e.g., recognizing objects from images, segmenting foreground in video frames), the DCNN accurately predicts perceptual similarity even near the discrimination threshold (Zhang et al. 2018).

In mimicry systems (and more generally in most communication systems) the relevant observer is not human. Are the performances of DCNNs at reproducing human perception also relevant to other animals? These performances are based on three characteristics (Kriegeskorte 2015). The first is the capacity of DCNNs to solve highlevel tasks (Zhang et al. 2018), which is shared by all animals. The second is their general architecture (a stack of convolution, activation, and pooling functions), which reproduces operations performed sequentially and iteratively by neurons in the different human visual areas (Güçlü and van Gerven 2015). Similar operations are also found, beyond humans, in at least all vertebrates (Renoult et al. 2019). The third is the structure of input data. In this study, we used RGB images that match human trichromatic vision (i.e., with three cone types). Other animals have different cone types (e.g., two in carnivores and four in birds) tuned to different wavelengths. How these differences affect DCNN models of perceptual space is a nearly unexplored avenue of research (but see Bergeron and Fuller 
2018). Yet indirect evidence suggests that the impact would be relatively minor. Indeed, studies of bird plumages that used both RGB images and bird-specific cone stimulations showed no qualitative differences (Dale et al. 2015; Miller et al. 2019). Accordingly, several authors have argued that RGB images provide good insight into ecology, evolution, and behavior in nonhuman species (Seddon et al. 2010; Bergeron and Fuller 2018). In addition, in our particular case the use of ranks to express relative resemblance makes our mimicry detection method insensitive to how precisely the similarity measure produced by DCNNs scales to similarity perceived by animals.

A potentially important limitation of RGB images, however, is that they eliminate wavebands to which humans - but not other vertebrates - are blind. The UV wavebands, in particular, may be problematic. Regarding our study, none of the main predators of snakes in the Western Palearctic use UV cues for foraging (Jacobs 2009; Lind et al. 2013, 2014). Mammalian predators are not able to see UV wavebands because they do not have UV cones (Jacobs 2009). Raptors have UV cones, but the previous suggestion that they use UV cues to detect prey (Viitala et al. 1995) is contentious, as their ocular media transmit UV only poorly (Lind et al. 2013). Thus, UV-based mimicry seems unlikely in Western Palearctic snakes.

Last, we analyzed images with minimum standardization (images were cropped to center the snake but not standardized for luminance, position, or background). Centering was necessary because of the small size of our training data set, but it could be omitted if a sufficient number of images were available. Although standardization removes irrelevant information from the data, we think that unstandardized images are ecologically more relevant. Indeed, predators experience substantial variation in perceptual conditions in relation to snake position, background, shading, light intensity, and color. This variation could affect the evolution of mimic phenotypes; for example, it has been suggested to explain imperfect mimicry (Kikuchi and Pfennig 2013). While RGB images from the internet may not precisely match the variation experienced by predators, drastically limiting all variation (by standardization) would make the data set unnatural. Standardized images would be nevertheless useful in other contexts (e.g., to determine which visual features contribute the most to deceive predators).

\section{Conclusions}

Our method rationalizes the search for mimicry evolution and as such is designed to select candidate mimics and models on the basis of a strong coincidence between resemblance and sympatry. It is complemented by biological observations that strengthen the case for mimicry (vs. other forms of convergent evolution). It is an improvement over pure naturalistic intuition because it is quantitative and has a clear rationale - a way to avoid the pitfalls of storytelling. By allowing a more comprehensive description of the diversity of mimicry, our methods could help us understand the selective forces acting on this defensive strategy but also generate new models to test hypotheses on mimicry developed in the literature. We propose that artificial intelligence has a great potential for the study of mimicry. Although there is still room for methodological improvement, DCNNs allow us to measure resemblance while accounting for the high dimensionality of visual phenotypes, simultaneously accounting for colors, textures, patterns, and proportions without requiring highly standardized data that may be difficult to collect for many species. Beyond mimicry, the ability to handle complex visual patterns makes DCNNs highly promising tools for modeling resemblance in general, with a broad range of applications in evolution and ecology.

\section{Acknowledgments}

We thank Matthieu Joron, Thomas Aubier, and Caroline Rose for their constructive and useful advice. All of the deep-learning analyses in this article benefited from the Montpellier Bioinformatics Biodiversity platform services. We also thank all of the photographers for sharing their photos.

\section{Statement of Authorship}

P.-A.C., P.D., T.d.S., and J.P.R. developed the question and the research approach. J.P.R. provided guidance for the DCNN analyses. P.G. provided pictures from his own collection, and T.d.S. collected the remaining pictures. T.d.S. wrote the Python script for the DCNN analyses and the $\mathrm{R}$ script for the analyses of the DCNN output. All authors contributed significant portions of original text to the manuscript, and all authors edited and provided comments on manuscript drafts.

\section{Data and Code Availability}

Data have been deposited in the Dryad Digital Repository (https://doi.org/10.5061/dryad.sj3tx961q; de Solan et al. 2019).

\section{Literature Cited}

Aubret, F., and A. Mangin. 2014. The snake hiss: potential acoustic mimicry in a viper-colubrid complex. Biological Journal of the Linnean Society 113:1107-1114.

Barlow, B. A., and D. Wiens. 1977. Host-parasite resemblance in Australian mistletoes: the case for cryptic mimicry. Evolution 31:69-84. 
Barnett, J. B., C. Michalis, N. E. Scott-Samuel, and I. C. Cuthill. 2018 Distance-dependent defensive coloration in the poison frog Dendrobates tinctorius, Dendrobatidae. Proceedings of the National Academy of Sciences of the USA 115:6416-6421.

Bates, H. W. 1862. Contributions to an insect fauna of the Amazon Valley. Lepidoptera: Heliconidae. Transactions of the Linnean Society of London 23:495-566.

Bergeron, Z. T., and R. C. Fuller. 2018. Using human vision to detect variation in avian coloration: how bad is it? American Naturalist 191:269-276.

Bosque, R. J., J. P. Lawrence, R. Buchholz, G. R. Colli, J. Heppard, and B. Noonan. 2018. Diversity of warning signal and social interaction influences the evolution of imperfect mimicry. Ecology and Evolution 8:7490-7499.

Brodie, E. D., and F. J. Janzen. 1995. Experimental studies of coral snake mimicry: generalized avoidance of ringed snake patterns by free-ranging avian predators. Functional Ecology 9:186-190

Carranza-Rojas, J., H. Goeau, P. Bonnet, E. Mata-Montero, and A. Joly. 2017. Going deeper in the automated identification of herbarium specimens. BMC Evolutionary Biology 17:181.

Cheney, K. L., and N. J. Marshall. 2009. Mimicry in coral reef fish: how accurate is this deception in terms of color and luminance? Behavioral Ecology 20:459-468.

Cichy, R. M., and D. Kaiser. 2019. Deep neural networks as scientific models. Trends in Cognitive Sciences 23:305-317.

Corcobado, G., M. E. Herberstein, and S. Pekár. 2016. The role of ultraviolet colour in the assessment of mimetic accuracy between Batesian mimics and their models: a case study using ant-mimicking spiders. Science of Nature 103:90-101.

Cuthill, I. C., and A. T. D. Bennett. 1993. Mimicry and the eye of the beholder. Proceedings of the Royal Society B 253:203-204.

Dale, J., C. J. Dey, K. Delhey, B. Kempenaers, and M. Valcu. 2015. The effects of life history and sexual selection on male and female plumage colouration. Nature 527:367-370.

Dalyac, A., M. Shanahan, and J. Kelly. 2014. Tackling class imbalance with deep convolutional neural networks. Imperial College, London.

Dalziell, A. H., and J. A. Welbergen. 2016. Mimicry for all modalities. Ecology Letters 19:609-619.

Darwin, C. 1887. Life and letters of Charles Darwin. J. Murray, London.

de Solan, T., J. P. Renoult, P. Geniez, P. David, and P.-A. Crochet. 2019. Data from: Looking for mimicry in a snake assemblage using deep learning. American Naturalist, Dryad Digital Repository, https://doi.org/10.5061/dryad.sj3tx961q.

Dittrich, W., F. Gilbert, P. Green, P. McGregor, and D. Grewcock. 1993. Imperfect mimicry: a pigeon's perspective. Proceedings of the Royal Society B 251:195-200.

Dixon, P. 2003. VEGAN, a package of R functions for community ecology. Journal of Vegetation Science 14:927-930.

Endler, J. A., G. L. Cole, and A. M. Kranz. 2018. Boundary strength analysis: combining colour pattern geometry and coloured patch visual properties for use in predicting behaviour and fitness. Methods in Ecology and Evolution 9:2334-2348.

Fisher, R. A. 1925. Statistical methods for research workers. Oliver \& Boyd, Edinburgh

Fuentes, A. F., S. Yoon, J. Lee, and D. S. Park. 2018. High-performance deep neural network-based tomato plant diseases and pests diag nosis system with refinement filter bank. Frontiers in Plant Science 9:1162.
Gans, C. 1961. Mimicry in procryptically colored snakes of the genus Dasypeltis. Evolution 15:72-91.

Geniez, P. 2015. Serpents d'Europe, d'Afrique du Nord et du MoyenOrient. Delachaux et Niestlé SA, Paris.

Gianoli, E., and F. Carrasco-Urra. 2014. Leaf mimicry in a climbing plant protects against herbivory. Current Biology 24:984-987.

Goodfellow, I., Y. Bengio, and A. Courville. 2016. Deep learning. MIT Press, Cambridge, MA.

Güçlü, U., and M. A. J. van Gerven. 2015. Deep neural networks reveal a gradient in the complexity of neural representations across the ventral stream. Journal of Neuroscience 35:1000510014.

Hansen, M. F., M. L. Smith, L. N. Smith, M. G. Salter, E. M. Baxter, M. Farish, and B. Grieve. 2018. Towards on-farm pig face recognition using convolutional neural networks. Computers in Industry 98:145-152.

Heatwole, H., and E. Davison. 1976. A review of caudal luring in snakes with notes on its occurrence in the Saharan sand viper, Cerastes vipera. Herpetologica 32:332-336.

Ishihara, K., S. Ishihara, M. Nagamachi, S. Hiramatsu, and H. Osaki. 2001. Age-related decline in color perception and difficulties with daily activities - measurement, questionnaire, optical and computer-graphics simulation studies. International Journal of Industrial Ergonomics 28:153-163.

Jacobs, G. H. 2009. Evolution of colour vision in mammals. Philosophical Transactions of the Royal Society B 364:2957-2967.

Johnstone, R. A. 1996. Multiple displays in animal communication: "backup signals" and "multiple messages." Philosophical Transactions of the Royal Society B 351:329-338.

Joshi, J., A. Prakash, and K. Kunte. 2017. Evolutionary assembly of communities in butterfly mimicry rings. American Naturalist 189:E58-E76.

Jozwik, K. M., N. Kriegeskorte, K. R. Storrs, and M. Mur. 2017. Deep convolutional neural networks outperform feature-based but not categorical models in explaining object similarity judgments. Frontiers in Psychology 8:1726-1754.

Ju, C., A. Bibaut, and M. J. van der Laan. 2018. The relative performance of ensemble methods with deep convolutional neural networks for image classification. Journal of Applied Statistics 45:2800-2818.

Kark, S., I. Warburg, and Y. L. Werner. 1997. Polymorphism in the snake Psammophis schokarion both sides of the desert edge in Israel and Sinai. Journal of Arid Environments 37:513-527.

Kikuchi, D. W., and D. W. Pfennig. 2010. High-model abundance may permit the gradual evolution of Batesian mimicry: an experimental test. Proceedings of the Royal Society B 277:10411048.

2013. Imperfect mimicry and the limits of natural selection. Quarterly Review of Biology 88:297-315.

Kim, J., A.-D. Nguyen, and S. Lee. 2019. Deep CNN-based blind image quality predictor. IEEE Transactions on Neural Networks and Learning Systems 30:11-24.

Kitamura, T., and M. Imafuku. 2015. Behavioural mimicry in flight path of Batesian intraspecific polymorphic butterfly Papilio polytes. Proceedings of the Royal Society B 282:20150483.

Kitayama, S., S. Duffy, T. Kawamura, and J. T. Larsen. 2003. Perceiving an object and its context in different cultures. Psychological Science 14:201-206.

Körschens, M., B. Barz, and J. Denzler. 2018. Towards automatic identification of elephants in the wild. arXiv, 1812.04418. 
Kriegeskorte, N. 2015. Deep neural networks: a new framework for modeling biological vision and brain information processing. Annual Review of Vision Science 1:417-446.

Kristiansen, E. B., S. D. Finkbeiner, R. I. Hill, L. Prusa, and S. P. Mullen. 2018. Testing the adaptive hypothesis of Batesian mimicry among hybridizing North American admiral butterflies. Evolution 72:1436-1448.

Kubilius, J., S. Bracci, and H. P. Op de Beeck. 2016. Deep neural networks as a computational model for human shape sensitivity. PLoS Computational Biology 12:e1004896.

Leviton, A. 1970. Review of the snakes of the genus Lytorhynchus Proceedings of the California Academy of Sciences 37:249-274.

Lind, O., M. Mitkus, P. Olsson, and A. Kelber. 2013. Ultraviolet sensitivity and colour vision in raptor foraging. Journal of Experimental Biology 216:1819-1826.

- 2014. Ultraviolet vision in birds: the importance of transparent eye media. Proceedings of the Royal Society B 281:20132209.

Mathis, A., P. Mamidanna, K. M. Cury, T. Abe, V. N. Murthy, M. W. Mathis, and M. Bethge. 2018. DeepLabCut: markerless pose estimation of user-defined body parts with deep learning. $\mathrm{Na}$ ture Neuroscience 21:1281-1289.

Mebert, K. 2011. Geographic variation of morphological characters in the dice snake (Natrix tessellata). Mertensiella 18:11-19.

Miller, E. T., G. M. Leighton, B. G. Freeman, A. C. Lees, and R. A. Ligon. 2019. Ecological and geographical overlap drive plumage evolution and mimicry in woodpeckers. Nature Communications 10:1602.

Mochida, K., W. Y. Zhang, and M. Toda. 2015. The function of body coloration of the hai coral snake Sinomicrurus japonicus boettgeri. Zoological Studies 54:33-39.

Mound, L. A., and P. Reynaud. 2005. Franklinothrips: a pantropical Thysanoptera genus of ant-mimicking obligate predators (Aeolothripidae). Zootaxa 864:1-16.

Nishikawa, H., T. Iijima, R. Kajitani, J. Yamaguchi, T. Ando, Y. Suzuki, S. Sugano, et al. 2015. A genetic mechanism for femalelimited Batesian mimicry in Papilio butterfly. Nature Genetics 47:405-409.

Norouzzadeh, M. S., A. Nguyen, M. Kosmala, A. Swanson, M. S. Palmer, C. Packer, and J. Clune. 2018. Automatically identifying, counting, and describing wild animals in camera-trap images with deep learning. Proceedings of the National Academy of Sciences of the USA 115:E5716-E5725.

Penney, H. D., C. Hassall, J. H. Skevington, K. R. Abbott, and T. N. Sherratt. 2012. A comparative analysis of the evolution of imperfect mimicry. Nature 483:461-464.

Pfennig, D. W., and S. P. Mullen. 2010. Mimics without models: causes and consequences of allopatry in Batesian mimicry complexes. Proceedings of the Royal Society B 277:2577-2585.

QGIS Development Team. 2013. Quantum geographic information system. Open Source Geospatial Foundation Project, Beaverton, OR

Rajpurohit, S., R. Parkash, and S. Ramniwas. 2008. Body melanization and its adaptive role in thermoregulation and tolerance against desiccating conditions in drosophilids. Entomological Research 38:49-60.

R Development Core Team. 2008. R: a language and environment for statistical computing. R Foundation for Statistical Computing, Vienna.

Renoult, J. P., B. Guyl, T. C. Mendelson, A. Percher, J. Dorignac, F. Geniet, and F. Molino. 2019. Modelling the perception of colour patterns in vertebrates with HMAX. bioRxiv, https://doi.org /10.1101/552307.
Saleh, M., and M. Sarhan. 2016. The egg-eating snake (Colubridae: Dasypeltis) of Faiyum, Egypt, with the description of a new species. Bulletin de la Société Herpétologique de France 160:25-48.

Sameen, M., and B. Pradhan. 2017. Severity prediction of traffic accidents with recurrent neural networks. Applied Sciences 7:476.

Santos, X., M. Vidal-García, J. C. Brito, S. Fahd, G. A. Llorente, F. Martínez-Freiría, X. Parellada, J. M. Pleguezuelos, and N. Sillero. 2014. Phylogeographic and environmental correlates support the cryptic function of the zigzag pattern in a European viper. Evolutionary Ecology 28:611-626.

Seddon, N., J. A. Tobias, M. Eaton, A. Ödeen, and B. E. Byers. 2010. Human vision can provide a valid proxy for avian perception of sexual dichromatism. Auk 127:283-292.

Shen, D., G. Wu, and H.-I. Suk. 2017. Deep learning in medical image analysis. Annual Review of Biomedical Engineering 19:221248.

Sherratt, T. N. 2002. The evolution of imperfect mimicry. Behavioral Ecology 13:821-826.

Stevens, M., C. P. Rong, and P. A. Todd. 2013. Colour change and camouflage in the horned ghost crab Ocypode ceratophthalmus. Biological Journal of the Linnean Society 109:257-270.

Taylor, C. H., T. Reader, and F. Gilbert. 2016. Hoverflies are imperfect mimics of wasp colouration. Evolutionary Ecology 30:567581.

Troscianko, J., J. Skelhorn, and M. Stevens. 2017. Quantifying camouflage: how to predict detectability from appearance. BMC Evolutionary Biology 17:7.

Turk, J. 1958. Observations on snakes and snake bite in the eastern Mediterranean area. Journal of the Royal Army Medical 104:63-71.

Valkonen, J. K., and J. Mappes. 2014. Resembling a viper: implications of mimicry for conservation of the endangered smooth snake. Conservation Biology 28:1568-1574.

Valkonen, J. K., M. Niskanen, M. Björklund, and J. Mappes. 2011a. Disruption or aposematism? significance of dorsal zigzag pattern of European vipers. Evolutionary Ecology 25:10471063.

Valkonen, J. K., O. Nokelainen, and J. Mappes. 2011b. Antipredatory function of head shape for vipers and their mimics. PLoS ONE 6:e22272.

Van Horn, G., O. Mac Aodha, Y. Song, Y. Cui, C. Sun, A. Shepard, H. Adam, P. Perona, and S. Belongie. 2018. The iNaturalist species classification and detection dataset. Pages 8769-8778 in Conference on Computer Vision and Pattern Recognition. IEEE, Piscataway, NJ.

Viitala, J., E. Korplmäki, P. Palokangas, and M. Koivula. 1995. Attraction of kestrels to vole scent marks visible in ultraviolet light. Nature 373:425-427.

Werner, Y. L. 1983. Behavioural triangulation of the head in three boigine snakes: possible cases of mimicry. Israel Journal of Zoology 32:205-228.

Werner, Y. L., and E. Frankenberg. 1982. Head triangulation in two colubrine snakes: probable behavioural reinforcement of Batesian mimicry. Israel Journal of Zoology 31:137-150.

Wilson, J. S., J. P. Jahner, M. L. Forister, E. S. Sheehan, K. A. Williams, and J. P. Pitts. 2015. North American velvet ants form one of the world's largest known Müllerian mimicry complexes. Current Biology 25:R704-R706.

Yamins, D. L. K., H. Hong, C. F. Cadieu, E. A. Solomon, D. Seibert, and J. J. DiCarlo. 2014. Performance-optimized hierarchical models predict neural responses in higher visual cortex. 
Proceedings of the National Academy of Sciences of the USA 111:8619-8624.

Zhang, R., P. Isola, A. A. Efros, E. Shechtman, and O. Wang. 2018. The unreasonable effectiveness of deep features as a perceptual metric. Pages 586-595 in Conference on Computer Vision and Pattern Recognition. IEEE, Piscataway, NJ.

Zhou, J., and O. G. Troyanskaya. 2015. Predicting effects of noncoding variants with deep learning-based sequence model. Nature Methods 12:931-934.

\section{References Cited Only in the Online Enhancements}

Barlow, A., K. Baker, C. R. Hendry, L. Peppin, T. Phelps, K. A Tolley, C. E. Wüster, and W. Wüster. 2013. Phylogeography of the widespread African puff adder (Bitis arietans) reveals multiple Pleistocene refugia in southern Africa. Molecular Ecology 22:1134-1157.

Chollet, F. 2015. Keras: deep learning library for theano and tensorflow. https://keras.io/.

- 2017. Xception: deep learning with depthwise separable convolutions. Pages 1800-1807 in Conference on Computer Vision and Pattern Recognition. IEEE, Piscataway, NJ.

Crochet, P.-A., J. B. Rasmussen, T. Wilms, P. Geniez, J.-F. Trape, and W. Böhme. 2008. Systematic status and correct nomen of the western North African cat snake: Telescopus tripolitanus (Werner, 1909) (Serpentes: Colubridae), with comments on the other taxa in the dhara-obtusus group. Zootaxa 1703:25-46.

Deng, J., W. Dong, R. Socher, L.-J. Li, K. Li, and L. Fei-Fei. 2009. ImageNet: a large-scale hierarchical image database. Pages 248 255 in IEEE Conference on Computer Vision and Pattern Recognition. IEEE, Piscataway, NJ.

Ferchaud, A.-L., S. Ursenbacher, M. Cheylan, L. Luiselli, D. Jelić, B. Halpern, Á. Major, et al. 2012. Phylogeography of the Vipera ursinii complex (Viperidae): mitochondrial markers reveal an east-west disjunction in the Palaearctic region. Journal of Biogeography 39:1836-1847.

Geniez, P. 2018. Snakes of Europe, North Africa and the Middle East: a photographic guide. Princeton University Press, Princeton, NJ.

Gonçalves, D. V., F. Martínez-Freiría, P.-A. Crochet, P. Geniez, S. Carranza, and J. C. Brito. 2018. The role of climatic cycles and trans-Saharan migration corridors in species diversification: biogeography of Psammophis schokari group in North Africa. Molecular Phylogenetics and Evolution 118:64-74.

IUCN (International Union for Conservation of Nature). 2018 The IUCN Red List of Threatened Species. Version 2018-2. https://www.iucnredlist.org/.

Kalyabina-Hauf, S., S. Schweiger, U. Joger, and W. Mayer. 2004. Phylogeny and systematics of adders (Vipera berus complex). Mertensiella 15:7-16.

Kelly, C. M. R., N. P. Barker, M. H. Villet, D. G. Broadley, and W. R. Branch. 2008. The snake family Psammophiidae (Reptilia: Serpentes): phylogenetics and species delimitation in the African sand snakes (Psammophis Boie, 1825) and allied genera. Molecular Phylogenetics and Evolution 47:1045-1060.

Kindler, C., M. Chèvre, S. Ursenbacher, W. Böhme, A. Hille, D. Jablonski, M. Vamberger, and U. Fritz. 2017. Hybridization patterns in two contact zones of grass snakes reveal a new Central European snake species. Scientific Reports 7:7378.
Kindler, C., P. de Pous, S. Carranza, M. Beddek, P. Geniez, and U. Fritz. 2018. Phylogeography of the Ibero-Maghrebian red-eyed grass snake (Natrix astreptophora). Organisms Diversity and Evolution 18:143-150.

Kyriazi, P., P. Kornilios, Z. T. Nagy, N. Poulakakis, Y. Kumlutaş, Ç. Ilgaz, A. Avc1, et al. 2013. Comparative phylogeography reveals distinct colonization patterns of Cretan snakes. Journal of Biogeography 40:1143-1155.

Mangiacotti, M., L. Limongi, M. Sannolo, R. Sacchi, M. A. L. Zuffi, and S. Scali. 2014. Head shape variation in eastern and western Montpellier snakes. Acta Herpetologica 9:167-177.

Mizsei, E., D. Jablonski, S. A. Roussos, M. Dimaki, Y. Ioannidis, G. Nilson, and Z. T. Nagy. 2017. Nuclear markers support the mitochondrial phylogeny of Vipera ursinii-renardi complex (Squamata: Viperidae) and species status for the Greek meadow viper. Zootaxa 4227:75.

Safaei-Mahroo, B., H. Ghaffari, H. Fahimi, S. Broomand, M. Yazdanian, E. Najafi Majd, S. S. Hosseinian Yousefkhani, et al. 2015 The herpetofauna of Iran: checklist of taxonomy, distribution and conservation status. Asian Herpetological Research 6:257-290.

Schätti, B., and A. Schmitz. 2006. Re-assessing Platyceps ventromaculatus (Gray, 1834) (Reptilia: Squamata: Colubrinae). Revue Suisse de Zoologie 113:747-768.

Schätti, B., F. Tillack, and C. Kucharzewski. 2014. Platyceps rhodorachis (JAN, 1863): a study of the racer genus Platyceps BLYTH, 1860 east of the Tigris (Reptilia: Squamata: Colubridae). Vertebrate Zoology 64:297-405.

Šmíd, J., B. Göçmen, P.-A. Crochet, J.-F. Trape, T. Mazuch, M. Uvizl, and Z. T. Nagy. 2019. Ancient diversification, biogeography, and the role of climatic niche evolution in the Old World cat snakes (Colubridae, Telescopus). Molecular Phylogenetics and Evolution 134:35-49.

Srivastava, N., G. Hinton, A. Krizhevsky, I. Sutskever, and R. Salakhutdinov. 2014. Dropout: a simple way to prevent neural networks from overfitting. Journal of Machine Learning Research 15:1929-1958.

Stümpel, N., M. Rajabizadeh, A. Avcı, W. Wüster, and U. Joger. 2016. Phylogeny and diversification of mountain vipers (Montivipera, Nilson et al., 2001) triggered by multiple Plio-Pleistocene refugia and high-mountain topography in the Near and Middle East. Molecular Phylogenetics and Evolution 101:336-351.

Taigman, Y., M. Yang, M. Ranzato, and L. Wolf. 2014. DeepFace: closing the gap to human-level performance in face verification. Pages 1701-1708 in Conference on Computer Vision and Pattern Recognition. IEEE, Piscataway, NJ.

Tamar, K., J. Šmíd, B. Göçmen, S. Meiri, and S. Carranza. 2016. An integrative systematic revision and biogeography of Rhynchocalamus snakes (Reptilia, Colubridae) with a description of a new species from Israel. PeerJ 4:e2769.

Trape, J.-F., and Y. Mané. 2006. Guide des serpents d'Afrique occidentale: savane et désert. IRD, Paris.

Trape, J.-F., and O. Mediannikov. 2016. Cinq serpents nouveaux du genre Boaedon Duméril, Bibron \& Duméril, 1854 (Serpentes: Lamprophiidae) en Afrique centrale. Bulletin de la Société Herpétologique de France 159:61-111.

Velo-Antón, G., R. Godinho, D. J. Harris, X. Santos, F. MartínezFreiria, S. Fahd, S. Larbes, et al. 2012. Deep evolutionary lineages in a Western Mediterranean snake (Vipera latastei/monticola group) and high genetic structuring in Southern Iberian populations. Molecular Phylogenetics and Evolution 65:965-973. 
Wade, E. 2001. Review of the false smooth snake genus Macroprotodon (Serpentes, Colubridae) in Algeria with a description of a new species. Bulletin of the Natural History Museum Zoology Series 67:85-107.

World Health Organization. 2010. Database of venomous snakes. https://apps.who.int/bloodproducts/snakeantivenoms/database /snakeframeset.html.
Zheng, J. 2009. Predicting software reliability with neural network ensembles. Expert Systems with Applications 36:21162122 .

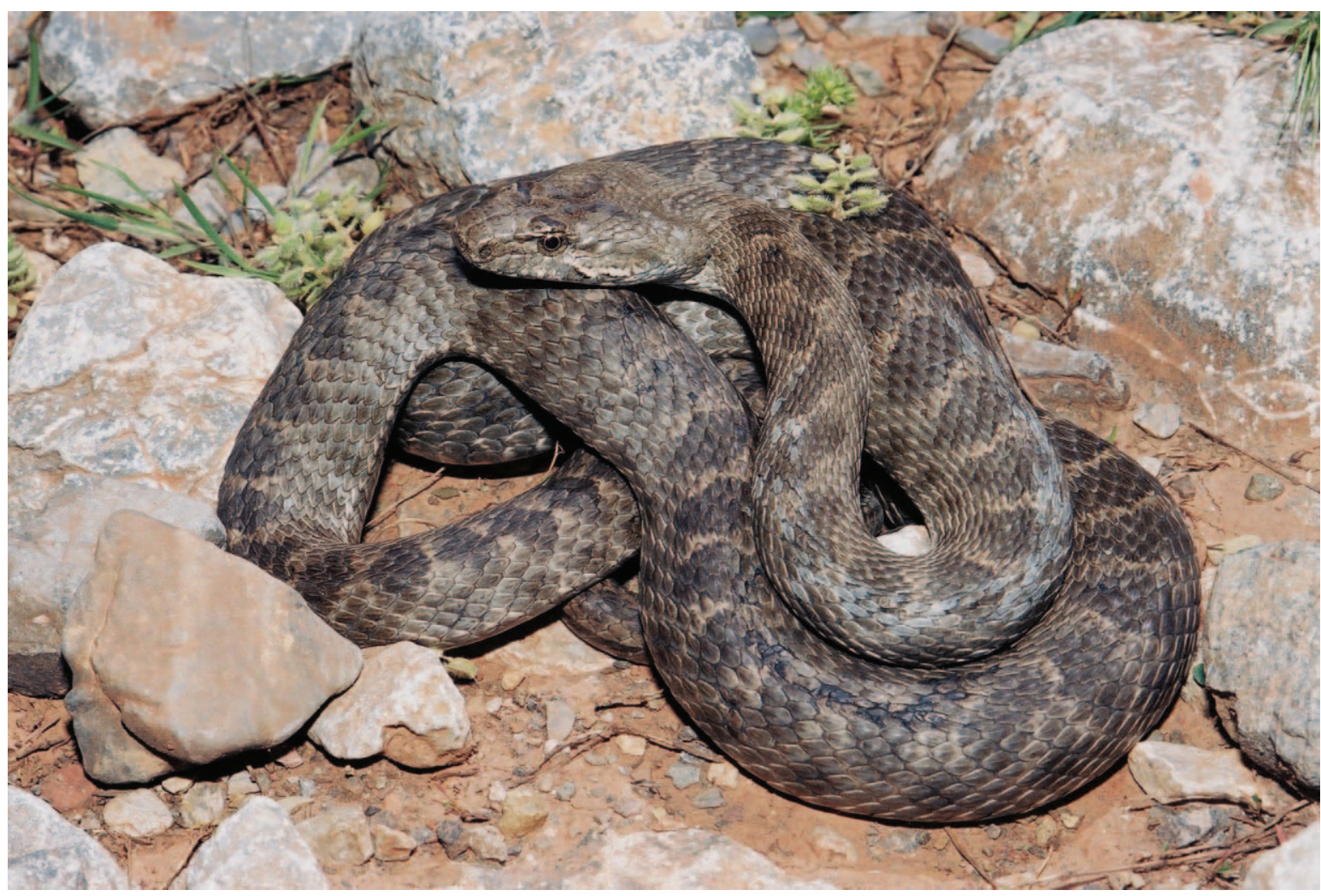

Hemorrhois nummifer. Photo credit: Philippe Geniez. 